\title{
Optimal settings and accuracy of indocyanine green fluorescence imaging for sentinel node biopsy in early gastric cancer
}

\author{
SHINICHI KINAMI, TOSHIO OONISHI, JUN FUJITA, YASUTO TOMITA, HIROSHI FUNAKI, \\ HIDETO FUJITA, YASUHARU NAKANO, NOBUHIKO UEDA and TAKEO KOSAKA \\ Department of Surgical Oncology, Kanazawa Medical University Hospital, Ishikawa 920-0293, Japan
}

Received March 21, 2015; Accepted April 8, 2016

DOI: $10.3892 / \mathrm{ol} .2016 .4492$

\begin{abstract}
Indocyanine green (ICG) fluorescence imaging represents a promising method for sentinel node (SN) biopsy in laparoscopic gastric surgery due to its signal stability. In the present study, the suitability and optimal settings of ICG fluorescence imaging for SN biopsy in early gastric cancer were determined. Patients with single primary superficial-type adenocarcinoma of the stomach, lesions $<5 \mathrm{~cm}$ in diameter, and no evident nodal metastasis and out of indication for endoscopic submucosal dissection were enrolled. The day prior to surgery, ICG solution was endoscopically injected into four quadrants of the submucosal layer of the tumor. The Photodynamic Eye was used to detect ICG fluorescence. Bright nodes were defined as clearly fluorescent nodes. A total of 72 patients were enrolled; 11 cases presented with metastasis, and of these, 10 could be diagnosed by bright node biopsy. The adequate concentration and injection volume of ICG was determined to be $50 \mu \mathrm{g} / \mathrm{ml}$ (x100) and $0.5 \mathrm{mlx} 4$ points, respectively. There was 1 false-negative case, and this was attributed to the failure of the frozen section diagnosis. These results suggested that ICG fluorescence imaging for SN biopsy in laparoscopic surgery for early gastric cancer is feasible. However, a weakness of ICG fluorescence imaging is the subjectivity of bright node evaluation.
\end{abstract}

\section{Introduction}

Gastric cancer patients who receive a standard gastrectomy with lymph node dissection can suffer from post-gastrectomy syndromes (1). As the symptoms associated with these syndromes can cause lifelong problems, a function-preserving gastrectomy with limited lymphadenectomy is recommended

Correspondence to: Dr Shinichi Kinami, Department of Surgical Oncology, Kanazawa Medical University Hospital, 1-1 Daigaku, Uchinada, Kahoku, Ishikawa 920-0293, Japan

E-mail:kinami@kanazawa-med.ac.jp

Key words: gastric cancer, sentinel lymph node biopsy, indocyanine green, fluorescence imaging to improve post-operative quality of life (1). A sentinel node (SN) biopsy is the most reliable method to date for the detection of node-negative cases, along with the application of an oncologically safe function-preserving gastrectomy.

The SN is defined as the node that directly receives lymphatic drainage from a primary tumor (2). Gastric cancer is one of the cancers in which the validity of the SN concept has been well investigated by numerous studies (3-17). Recently, a prospective multicenter trial demonstrated the feasibility of SN biopsy in gastric cancer (3). However, a number of unresolved issues remain regarding the clinical use of SN biopsy for gastric cancer, including identification of an adequate tracer for laparoscopic gastrectomy $(3,11,12)$. The dual-tracer method with blue dye and radioactive colloid appears to be a temporary standard procedure in gastric cancer. However, blue dye deteriorates quickly, and radioactive colloid exhibits a shine-through effect during $\gamma$ probe detection of hot nodes. These weaknesses limit their usefulness for laparoscopic SN biopsy.

One tracer candidate being evaluated for laparoscopic gastrectomy is indocyanine green (ICG). With the recent invention of useful devices such as the Photodynamic Eye (PDE) and Hyper Medical Eye System, ICG fluorescence imaging for SN biopsy has been widely used in various cancer surgeries (18-27). ICG fluorescence imaging has unique characteristics, including an extra-high sensitivity $(26,28)$ and signal stability $(23,26,28)$. However, the optimal settings of ICG fluorescence imaging for SN biopsy, such as ICG dose and concentration, injection timing and biopsy technique, have not been determined. Furthermore, little is known regarding the weaknesses of this technique.

The present study determined whether ICG fluorescence imaging is a suitable method for SN biopsy in laparoscopic gastrectomy. In addition, the optimal setting and weaknesses of this method were investigated.

\section{Patients and methods}

Patients. Between October 2009 and December 2014, 72 patients with histologically confirmed clinical type 0 (superficial-type) adenocarcinoma of the stomach (29), single primary lesions $<5 \mathrm{~cm}$ in diameter, no previous history of chemotherapy, and no distant metastasis (cM0) or evident nodal metastasis (cNO) and out of indication of endoscopic 
submucosal dissection were enrolled in the study. Clinical staging was determined by pre-operative endoscopy and multidetector-row computed tomography. All patients enrolled in the study were pre-operatively registered in the data center in Kanazawa Medical University Hospital (Ishikawa, Japan).

All patients provided written informed consent. This study was approved by the Ethics Committee of Kanazawa Medical University and conducted in accordance with the Good Clinical Practice guidelines and Declaration of Helsinki.

SN mapping procedure. The PDE (Hamamatsu Photonics Co., Ltd., Hamamatsu, Shizuoka, Japan) or PDE neo was used to detect ICG fluorescence. PDE neo is a newer version of the PDE with similar sensor sensitivity. A single, well-trained surgeon performed all tracer injections, surgeries, mappings and nodal harvestings to avoid disruption of the learning phase. During the study period, laparoscope-assisted gastrectomy was categorized as clinical research in the Gastric Cancer Treatment Guidelines of the Japanese Gastric Cancer Association (30). Therefore, the surgical approach adopted (open or laparoscope-assisted) was based on patient decision under informed consent.

The day prior to the surgery, ICG solution was endoscopically injected into four quadrants of the submucosal layer of the primary lesion using an endoscopic puncture needle. The gastrocolic ligament was divided to visualize all possible directions of lymphatic flow from the stomach. In open surgery cases, the PDE or PDE neo was then used to detect ICG fluorescence. For laparoscope-assisted surgery cases, the stomach and perigastric lymph nodes were pulled up and exposed from a 4-6 cm median upper abdominal incision, and ICG fluorescence in the lymphatic vessels and lymph nodes was detected with the PDE or PDE neo in the same manner as used for open surgery. Bright nodes were defined as clearly fluorescent nodes. The bright nodes were believed to contain the SNs. The number and direction of the lymphatic basins were observed and recorded intraoperatively by the surgeon. Lymphatic basins were defined as lymphatic zones divided by the stream of fluorescent lymphatic canals. The proximal border was the fatty tissue attached to the stomach wall, and the distal border was the front of the bright node most distal from the stomach $(4,5)$.

SN biopsy procedures. The feasibility of ICG imaging for SN biopsy was evaluated from the beginning of the study until December 2012 in 42 sequential patients who received standard gastrectomy with lymph node dissection up to D1+ or D2 according to the Gastric Cancer Treatment Guidelines (30). After noting the state of the lymphatic basin, a standard gastrectomy with lymph node dissection was conducted. Bright node detection was performed post-operatively in the initial 16 cases. Subsequent to surgery, all dissected nodes were harvested and analyzed for fluorescence using the PDE. Intraoperative bright nodes biopsy was performed in the other 26 cases. Following stomach removal and nodal dissection, but prior to reconstruction, bright nodes were detected and harvested at the surgical field and sent for pathological diagnosis of the intraoperative frozen section.
After the feasibility phase of the study, the clinical application of ICG SN mapping in guiding limited surgery was evaluated from January 2013. In this phase of the study, patients received function-preserving gastrectomy with lymphatic basin dissection. Function-preserving gastrectomies included pylorus-preserving gastrectomy, proximal gastrectomy, segmental gastrectomy and local resection of the stomach. Lymphatic basin dissection is a selective lymphadenectomy to dissect en bloc the lymphatic basins that contain lymph nodes and lymphatic vessels $(4,5)$. Following resection, the bright nodes were detected and harvested at the surgical field and sent for pathological diagnosis of the intraoperative frozen section. The surgeon performed the reconstruction while waiting for the pathology results. For bright nodes without metastasis, the surgical team continued the reconstruction and finished the procedure. For cases with nodal metastasis, the surgery was converted to a standard D2 gastrectomy (distal gastrectomy or total gastrectomy).

Post-operatively, all dissected nodes were reexamined for fluorescence using the PDE. Therefore, there were two types of bright nodes, nodes detected intraoperatively and those detected post-operatively.

Pathological examination. The frozen section and permanent diagnoses of nodal metastasis were made on a plane of the maximal dimension containing the hilus of the node according to the Japanese Classification of Gastric Carcinoma (29). The intraoperative frozen section diagnosis was followed by an additional permanent pathological diagnosis following node fixation in formaldehyde. Nodal metastasis was defined as the presence of tumor cells, tumor cell clusters or tumor cell nests within the lymph node, as detected by hematoxylin-eosin staining. Size was not included as a criterion for nodal metastasis and therefore, nodal metastasis included micrometastases and isolated tumor cells. The regional lymph nodes of the stomach were classified into stations numbered according to the Japanese Classification of Gastric Carcinoma (29).

Determination of the adequate concentration and injection volume of ICG. To determine the adequate ICG concentration for SN biopsy, $0.5 \mathrm{mlx} 4$ points of $2.5 \mathrm{mg} / \mathrm{ml}, 125 \mu \mathrm{g} / \mathrm{ml}$, $50 \mu \mathrm{g} / \mathrm{ml}$ and $5 \mu \mathrm{g} / \mathrm{ml}$ ICG were preliminarily examined in one or two cases. After identifying the adequate concentration, ICG injection volumes of $0.5 \mathrm{mlx} 4$ points and $0.2 \mathrm{mlx} 4$ points were evaluated.

\section{Results}

Patients. A total of 72 patients were enrolled in the study. This consisted of 42 patients for the feasibility phase and 30 patients for the clinical application phase. Patient characteristics are shown in Table I. A laparoscopic approach was performed in $55.6 \%$ of patients. No serious allergic reactions were observed following tracer injection. Intraoperative and post-operative adverse effects and complications associated with the SN biopsy procedures were not observed.

SN biopsy results. Bright nodes and lymphatic basins were detected in all cases (Fig. 1); therefore, the detection rate 
Table I. Patient characteristics.

Characteristic

Feasibility phase $(n=42)$

Application phase $(n=30)$

Median Age (range), years

$68(47-82)$

$71(54-84)$

Male/female, $n$

$26 / 16$

$18 / 12$

Tumor location, $\mathrm{n}$

Upper third G/A/L/P

$1 / 0 / 3 / 1$

$1 / 0 / 8 / 2$

Middle third G/A/L/P

$3 / 3 / 15 / 2$

$4 / 2 / 4 / 2$

Lower third G/A/L/P

$6 / 2 / 6 / 0$

$0 / 2 / 3 / 2$

Pathological depth of invasion

T1a(M)/T1b(SM)/T2(MP)/T3 (SS), n

$16 / 17 / 8 / 1$

$13 / 14 / 2 / 1$

Median tumor size post-gastrectomy (range), mm

$28(10-55)$

$27(8-65)$

Macroscopic findings, $n$

Elevated/depressed

$10 / 20$

Surgical approach, $\mathrm{n}$

Open/laparoscopic

$15 / 27$

$17 / 13$

A, anterior wall; G, greater curvature; L, lesser curvature; M, mucosal; MP, proper muscle; P, posterior wall; SM, submucosal; SS, subserosal.

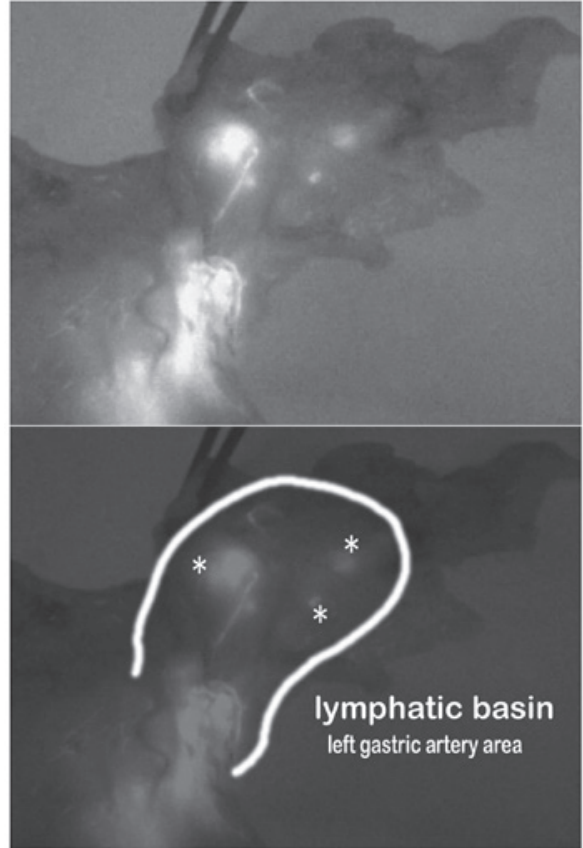

Figure 1. Bright nodes and lymphatic basin detection by indocyanine green fluorescence imaging with the Photodynamic Eye. An intraoperative image obtained after distal partial gastrectomy with standard lymph node dissection up to D2 is shown. The tumor was located at the lesser curvature of the middle third of the stomach. Fluorescence was detected in the lymphatics and lymph nodes. In this case, the lymphatic basin was the left gastric artery area, and all bright nodes $(*)$ were no. 3 .

of ICG fluorescence imaging was $100.0 \%$. The sensitivity, specificity and accuracy of ICG fluorescence SN mapping were 90.1, 100.0 and 98.6\%, respectively (Table II).

In total, 11 cases presented with metastasis. In the feasibility case series $(n=42), 7$ cases presented with lymph node metastasis, and all cases were able to be diagnosed using bright node biopsy. Of these 7 cases, 4 were diagnosed post-operatively in the permanent pathological diagnosis and 3 were diagnosed intraoperatively in the frozen section diagnosis. In the clinical application series, 4 cases presented with lymph node metastasis, and of these, 3 cases were able to be diagnosed by bright node biopsy. Thus, 1 case with nodal metastasis failed to be diagnosed intraoperatively.

Details of the false-negative case. Intraoperative bright node biopsy resulted in 1 false-negative case. The false-negative result occurred in a 72-year-old female with a 20 -mm IIc-type tumor with an ulcer scar located on the greater curvature near the posterior wall of the middle third of the stomach. The final pathological diagnosis was non-solid type poorly-differentiated adenocarcinoma, submucosal cancer with submucosal invasion of $>500 \mu \mathrm{m}$ from the muscularis mucosa, marked lymphatic invasion and minimal venous invasion, based on the Japanese Classification of Gastric Carcinoma (29). The lymphatic basins were the left gastric artery and right gastroepiploic artery areas, and a laparoscopic pylorus-preserving gastrectomy with lymphatic basin dissection was performed. The station numbers of the dissected nodes were nos. 1, 3a, 4d, 6 and 7. A total of 8 bright nodes were detected intraoperatively and of these, 2 nodes were no. 3 a, 1 node was no. 7 and 5 nodes were no. $4 \mathrm{~d}$. Post-surgery, at the time of nodal harvesting, 2 bright nodes were found at no. $3 \mathrm{a}$ and $4 \mathrm{~d}$. The no. $3 \mathrm{a}$ node exhibited weak fluorescence and appeared to be a secondary node. The no. $4 \mathrm{~d}$ node exhibited strong fluorescence and appeared to be a node that failed to be detected intraoperatively due to the shine-through effect.

A total of 2 nodal metastases were present and were not the bright nodes detected intraoperatively. The involved nodes were both no. $4 \mathrm{~d}$ and were located near the tumor. One of the nodes was the bright node detected after surgery and was classified as a micrometastasis. The other metastasis was a non-bright node located near the bright node and was 
Table II. Bright nodes biopsy results for the nodal metastasis cases in the feasibility $(n=42)$ and clinical $(n=30)$ phases.

Nodal status of all

dissected nodes

Status of sentinel

node biopsy

Metastasis No metastasis

Feasibility phase, $\mathrm{n}$

Bright node biopsy

pathological diagnosis

Metastasis 7

No metastasis 0 35

Clinical phase, $\mathrm{n}$

Bright node biopsy

frozen section diagnosis

Metastasis

No metastasis 3

1 26

Sensitivity \% (n/total n)

$90.1(10 / 11)$

Accuracy \% (n/total n)

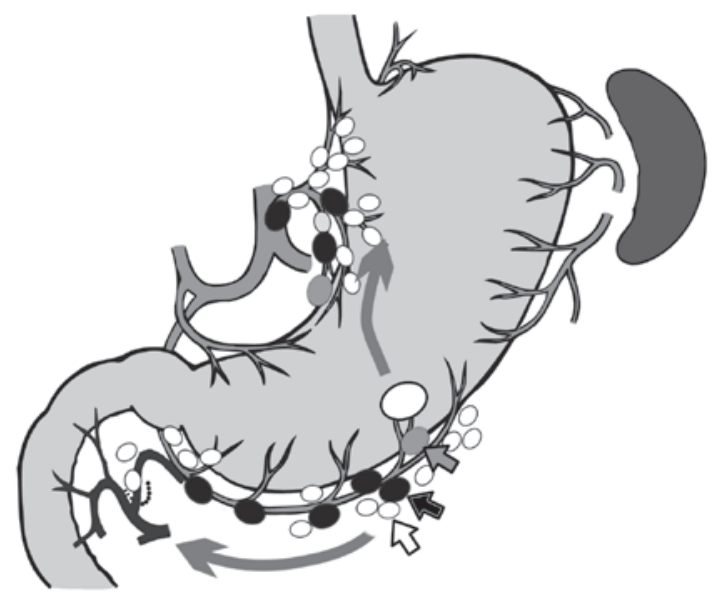

Figure 2. Distribution pattern of dissected and metastatic nodes in the false-negative case. The tumor was located on the greater curvature near the posterior wall of the middle third of the stomach (white circle). The lymphatic basins were the left gastric artery and right gastroepiploic artery areas (large arrows). A total of 8 bright nodes were detected intraoperatively (black nodes), 2 bright nodes were found post-operatively at the time of nodal harvesting (gray nodes), and 2 nodal metastases were present and were not the bright nodes detected intraoperatively; 1 of the nodes was the bright node detected post-operatively and was classified as a micrometastasis (gray arrow). The other metastasis was classified as isolated tumor cells (white arrow). Another metastasis classified as isolated tumor cells was diagnosed in deep cuttings of the bright node detected intraoperatively (black arrow). The small white circles outside the stomach represent the regional lymph nodes dissected during surgery.

classified as isolated tumor cells. After the metastases were diagnosed, deep cuttings and other slices of the paraffin blocks of the intraoperatively detected bright nodes were made and revealed the presence of isolated tumor cells in one of the no. $4 \mathrm{~d}$ bright nodes (Fig. 2). Lymph node dissection up to D1 was performed, and the dissection margin appeared

to be cancer free. Therefore, this case was considered to be a successful curative resection. The patient has since been closely followed up and has shown no evidence of recurrence to date.

Adequate concentration and injection volume of ICG. The adequate concentration of ICG was determined in the initial 8 cases, which were all node-negative. Table III shows the association between the number of bright nodes and ICG concentration. The cases were arranged in order of occurrence to show the decision process. At an ICG concentration of $5 \mu \mathrm{g} / \mathrm{ml}(\mathrm{x} 1,000)$, bright lymphatics and bright nodes were detected despite the weak fluorescence. Therefore, the adequate concentration of ICG was determined to be $50 \mu \mathrm{g} / \mathrm{ml}(\mathrm{x} 100)$.

Next, the adequate injection volume of $50 \mu \mathrm{g} / \mathrm{ml} \mathrm{ICG}$ was determined. A total of 15 and 19 sequential patients were injected with $0.5 \mathrm{mlx} 4$ points and $0.2 \mathrm{mlx} 4$ points of $50 \mu \mathrm{g} / \mathrm{ml}$ ICG, respectively. The visualization of bright nodes and bright lymphatics was quite similar between the two groups. The median number of bright nodes was not significantly different between the two groups, with 6 (range, 3-11) in the $0.5 \mathrm{ml}$ group and 6 (range, 2-7) in the $0.2 \mathrm{ml}$ group. Therefore, the adequate injection volume of ICG solution was determined to be $0.5 \mathrm{mlx} 4$ points due to its technical reliability.

Number of bright nodes. In the present study, 68 patients were injected with $50 \mu \mathrm{g} / \mathrm{ml}$ ICG solution. Of these patients, bright nodes were detected post-operatively only in 14 patients, but both intraoperatively and post-operatively in 54 patients. The median number of bright nodes was 5 (range, 4-11) and 6 (range, 2-9) in the post-operative only and intraoperative cases, respectively. In 27 out of the 54 intraoperative cases $(50.0 \%)$, bright nodes left behind were also detected post-operatively. The total number of nodes in these cases was 49 , and the median number of nodes per each case was 1 (range, $0-4$ ) (Table IV). Of these 49 nodes, 31 (63\%) exhibited weak fluorescence and 18 (37\%) exhibited strong fluorescence. Almost all strongly fluorescent nodes were small nodes $<5 \mathrm{~mm}$ in diameter, located near the serosa of the stomach, covered by fat, and were difficult to detect intraoperatively due to the shine-through effect of the serosal bright spots. By contrast, the 31 nodes with weak fluorescence were located far from the tumor and appeared to be secondary nodes.

Distribution of metastatic nodes. All dissected nodes in this study were divided into the following three groups: Bright nodes, non-bright nodes within the lymphatic basin and non-bright nodes outside the lymphatic basin. A total of 11 cases $(15.3 \%)$ presented with nodal metastasis. Lymph node dissection up to D2 was performed in 10 of these cases, while the other case was the false-negative intraoperative case. Therefore, the distribution of metastatic nodes within and outside (up to D2) the lymphatic basins was well examined in these cases. The total number of metastatic nodes was 35 and of these, $22(62.9 \%)$ were bright nodes, $12(34.3 \%)$ were non-bright nodes within the basin and 1 (2.9\%) was a non-bright node outside the basin. 
Table III. Association between the number of bright nodes and indocyanine green concentration.

\begin{tabular}{llrr}
\hline Case no. & Concentration & Volume & No. of bright nodes \\
\hline 1 & x2 $(2.5 \mathrm{mg} / \mathrm{ml})$ & $0.5 \mathrm{mlx} 4$ & 17 \\
2 & $\mathrm{x} 40(125 \mu \mathrm{g} / \mathrm{ml})$ & $0.5 \mathrm{mlx} 4$ & 8 \\
3 & $\mathrm{x} 100(50 \mu \mathrm{g} / \mathrm{ml})$ & $0.5 \mathrm{mlx} 4$ & 4 \\
4 & $\mathrm{x} 100(50 \mu \mathrm{g} / \mathrm{ml})$ & $0.5 \mathrm{mlx} 4$ & 6 \\
5 & $\mathrm{x} 40(125 \mu \mathrm{g} / \mathrm{ml})$ & $0.5 \mathrm{mlx} 4$ & 8 \\
6 & $\mathrm{x} 100(50 \mu \mathrm{g} / \mathrm{ml})$ & $0.5 \mathrm{mlx} 4$ & 5 \\
7 & $\mathrm{x} 100(50 \mu \mathrm{g} / \mathrm{ml})$ & $0.5 \mathrm{mlx} 4$ & 7 \\
8 & $\mathrm{x} 1000(5 \mu \mathrm{g} / \mathrm{ml})$ & $0.5 \mathrm{mlx} 4$ & 3 \\
$9-23$ & $\mathrm{x} 100(50 \mu \mathrm{g} / \mathrm{ml})$ & $0.5 \mathrm{mlx} 4$ & $6(3-11)$ \\
$24-42$ & $\mathrm{x} 100(50 \mu \mathrm{g} / \mathrm{ml})$ & $0.2 \mathrm{mlx} 4$ & $6(2-7)$ \\
\hline
\end{tabular}

Table IV. Association between the number of bright nodes and timing of nodal detection.

\begin{tabular}{lcc}
\hline Detection of bright nodes & $\begin{array}{c}\text { No. of } \\
\text { cases }\end{array}$ & $\begin{array}{c}\text { Median no. } \\
\text { of bright } \\
\text { nodes (range) }\end{array}$ \\
\hline $\begin{array}{l}\text { Post-operative only } \\
\text { Intraoperative and post-operative }\end{array}$ & 54 & $5(4-11)$ \\
$\quad$ Intraoperative & & $6(2-9)$ \\
Post-operative (pick-up failure) & & $1(0-4)$ \\
\hline
\end{tabular}

Among the 11 nodal metastasis cases, all metastatic nodes were bright nodes in 5 cases, and metastatic nodes were bright and non-bright nodes within the basins in 5 cases. In the remaining case, the metastatic node was located not only within the basin, but also outside the basin. This case involved a 62-year-old male whose tumor was located at the greater curvature of the lower third of the stomach. The macroscopic findings revealed a IIc tumor measuring 40×30 mm, and the pathological findings were moderately differentiated tubular adenocarcinoma, tumor invasion of the muscularis propria, tumor with mild infiltrating growth and mildly indistinct border with the surrounding tissue, and moderate lymphatic and venous invasion, based on the Japanese Classification of Gastric Carcinoma (29). The lymphatic basin was the right gastroepiploic artery area, and the 5 bright nodes were no. $4 \mathrm{~d}$. The pre-operative diagnosis based on helical computed tomography was cN0, even though the patient had an evident macroscopic metastatic node at station no. 6. The patient presented with 7 metastatic nodes consisting of 2 bright nodes, 4 non-bright nodes within the basin and 1 non-bright node outside the basin (no. 7). This case was believed to be an out of indication for SN biopsy due to the presence of macrometastases and the ease of metastasis diagnosis.

Clinical application of SN biopsy with ICG. In total, 30 cases were used to evaluate the usefulness of ICG fluorescent SN navigation to guide limited surgery. The specifics of the surgeries are shown in Table V. A function-preserving curative gastrectomy with lymphatic basin dissection (limited surgeries beyond the guideline procedures) was performed in 20 out of the 30 cases $(66.7 \%)$. No evidence of recurrence has been observed in any of these cases to date.

\section{Discussion}

The validity of the SN concept remains under debate even in gastric cancer. Over past decades, the application of SN mapping in gastric cancer, which has a relatively complicated lymphatic flow, has been a controversial issue (31). However, more recent prospective studies have successfully demonstrated the utility of SN biopsy in gastric cancer (6-10). Most recently, two large-scale nationwide multicenter prospective studies, the JCOG0302 study (11) and the SN navigation surgery (SNNS) study by Kitagawa et al (3), were conducted to evaluate SN mapping in gastric cancer. The JCOG0302 study provided evidence to support the use of this technique in gastric cancer. However, the JCOG0302 trial was terminated due to the high proportion of false-negative results obtained in the intraoperative histological examination (11). The JCOG0302 study results were affected by the intraoperative $\mathrm{SN}$ detection technique and intraoperative diagnosis of nodal metastasis. By contrast, the SNNS study was specifically designed to verify the SN concept in gastric cancer (3).

SN biopsy has been considered to exhibit two main roles in various cancers, namely, ultrastaging and the guidance of lymph node dissection omission. In malignant melanoma and colorectal cancer management, SN biopsy is primarily used for ultrastaging (2,32). By contrast, SN biopsy is mainly used to guide lymph node dissection omission in breast cancer surgery (33). For gastric cancer, clinical studies have primarily focused on the application of SN biopsy in guiding lymph node dissection omission. However, lymph node dissection omission deserves circumspection, as standard lymph node dissection is associated with an improved prognosis in gastric cancer patients (34). Furthermore, in contrast to breast cancer, reoperation for additional nodal dissection is rarely performed in gastric cancer (5).

SN biopsy is a complex multistep surgical technique. This technique requires a suitable limitation of the indication, the selection of an adequate tracer, a proper tracer injection method, the objective detection of tracer uptake 
Table V. Surgeries performed in the feasibility and clinical phases.

A, Feasibility phase $(n=42)$

\begin{tabular}{lcr}
\hline Type & Procedure & No. \\
\hline Meta(+) & DG & 2 \\
& LADG & 4 \\
& PG & 1 \\
Meta(-) & DG & 6 \\
& LADG & 10 \\
& PPG & 2 \\
& LAPPG & 13 \\
& PG & 3 \\
& TG & 1 \\
\hline
\end{tabular}

$B$, Clinical phase $(n=30)^{a}$

\begin{tabular}{lcc}
\hline Type & Procedure & No. \\
\hline Meta(+) & LADG & 3 \\
Meta(-) & LAPPG & 1 \\
& DG & 1 \\
& LADG & 2 \\
PPG & 2 \\
LAPPG & 1 \\
& Limited DG & $\mathbf{1}$ \\
& LA-limited DG & $\mathbf{1}$ \\
SGG & $\mathbf{2}$ \\
LASG & $\mathbf{1}$ \\
& Limited PG & $\mathbf{6}$ \\
Local resection & $\mathbf{4}$ \\
& LA-local resection & $\mathbf{5}$
\end{tabular}

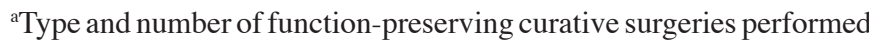
in the clinical phase are shown in bold. DG, distal gastrectomy; LA, laparoscope-assisted; meta(+), lymph node metastasis-positive; meta(-), lymph node metastasis-negative; PG, proximal gastrectomy; PPG, pylorus-preserving gastrectomy; SG, segmental gastrectomy; TG, total gastrectomy.

by the nodes, a reliable biopsy technique for the nodes that show tracer uptake and the precise intraoperative detection (micrometastasis level) of nodal metastasis. A number of studies have provided recommendations regarding the suitable indication and tracer injection method for SN biopsy in gastric cancer. In a meta-analysis of SN biopsy for gastric cancer by Wang et al (12), early T stage, submucosal injection method, combined tracers, conventional open surgery and immunohistochemistry application were demonstrated to be associated with a higher rate of $\mathrm{SN}$ identification and higher sensitivity.

The selection of an adequate tracer for SN mapping in gastric cancer has been an important issue $(3,11,12)$. A temporary standard is combination mapping with technetium- $99 \mathrm{~m}$ tin colloid and isosulfan blue. However, blue dye deteriorates quickly, and radioactive colloids exhibit a shine-through effect during $\gamma$ probe detection of hot nodes in the surgical field. We believe that combination mapping is not suitable for laparoscopic gastrectomy.

ICG is a promising tracer candidate for laparoscopic gastrectomy $(14,15)$. ICG absorbs light in the near-infrared range, with maximum absorption at the $800-\mathrm{nm}$ wavelength. ICG also emits maximal fluorescence at the 840-nm wavelength upon binding to plasma proteins. Nimura et al (13) developed an infrared ray electronic endoscopy system for ICG detection. Kusano et al (14) demonstrated the high sensitivity of this ICG fluorescence imaging system for SN mapping in gastric cancer. The advantages of ICG fluorescence imaging are the low cost of ICG, lower equipment costs compared with radioactive tracers, no requirement for radioactivity, a lower frequency of allergic reactions compared with blue dye, the ability to detect bright nodes under thick adipose tissue (14), clear visualization, easy detection of bright nodes and lymphatic canals compared with the naked eye or infrared ray imaging (23), an extra high sensitivity capable of detecting minute concentrations of ICG $(26,28)$ and signal stability $(23,26,28)$. Signal stability is a unique characteristic of ICG fluorescence imaging (15). The stability of ICG fluorescence imaging allowed us to successfully use pre-operative injections for SN detection. However, ICG fluorescence imaging also has certain clear disadvantages, such as the requirement for fluorescence detection equipment and a potential shine-through effect. The high sensitivity of this technique is also a disadvantage, as it can cause secondary node contamination of bright nodes.

Based on our experience, $50 \mu \mathrm{g} / \mathrm{ml} \mathrm{ICG}$ is adequate for detection by the PDE. At this concentration, the median number of bright nodes was 6 in the present study, which is similar to the number obtained in our previous dye method study (4). Furthermore, good results in sensitivity and accuracy were achieved regardless of surgical approach, i.e., laparoscopic or open surgery. A $50 \mu \mathrm{g} / \mathrm{ml}$ ICG concentration was also used with the HyperEye Medical System in the study by Yoshida et al (26). Nevertheless, the proper concentration of ICG should be reexamined if other detection devices are used.

SN biopsy for gastric cancer also requires a reliable bright node biopsy technique and objective bright node detection. With regard to biopsy technique, the lymphatic basin dissection method must be used even for ICG fluorescence imaging. Unlike other cancers, lymphatic basin dissection is a standard technique in gastric cancer $(4,5)$. The lymphatic basins are believed to be the primary lymphatic drainage area in each patient, and those individuals with gastric cancer often exhibited two or three basins $(3,4,5,16,17)$. Lymphatic basin dissection is superior to the ordinary pick-up method not only in terms of preventing missed bright nodes, but also with regard to oncological safety, as it complements the intraoperative pathological diagnosis by serving as a backup dissection $(5,16,17)$. In contrast to the biopsy technique, objective bright node detection remains an unresolved issue of SN biopsy. In the present study, a few bright nodes were detected post-operatively in the intraoperative cases. These nodes were left behind in the lymphatic basin following the intraoperative biopsy of the bright nodes and were of two types: Strongly bright nodes that were missed in the intraoperative biopsy due to the shine-through effect of 
the primary lesion, and weakly bright nodes that were believed to be secondary nodes. The shine-through effect could be eliminated by using the lymphatic basin dissection method and performing bright node detection after dissection of the basin from the gastric wall. However, secondary node contamination is problematic, as true SNs are difficult to distinguish from secondary nodes. Novel fluorescence agents have already been developed that have fluorescence and colloid particle characteristics (35-39). These novel agents would only detect fluorescent SNs and not secondary nodes, and therefore, would be the most potentially useful for conducting a laparoscopic SN biopsy in gastric cancer. Furthermore, they may potentially be used alone as a standard tracer instead of in combination with other SN mapping tracers.

The most important issue in performing SN biopsy is the intraoperative diagnosis of nodal metastasis. Frozen section diagnosis with rapid hematoxylin-eosin staining can potentially result in misdiagnosis. In the present series, only one false-negative case occurred due to the failure of the frozen section diagnosis. To solve this issue and establish oncological safety, molecular methods to diagnose nodal metastasis, such as reverse transcriptase-polymerase chain reaction and one-step nucleic acid amplification assays, should be developed for clinical use $(40,41)$.

In conclusion, ICG fluorescence imaging for SN biopsy is feasible in open and laparoscopic surgery for early gastric cancer. The optimal tracer setting to use with the PDE is an endoscopic submucosal injection of $0.5 \mathrm{ml}$ of $50 \mu \mathrm{g} / \mathrm{ml} \mathrm{ICG}$ at four points surrounding the tumor the day prior to surgery. The distribution of metastatic nodes in ICG fluorescence mapping is consistent with the basin theory $(3,4,5)$. Therefore, ICG fluorescence SN biopsy could be used to guide lymph node dissection omission, thereby allowing for function-preserving gastrectomy with lymphatic basin dissection. A weakness of ICG fluorescence imaging is the subjectivity of SN evaluation and potential secondary node contamination. The present study findings also suggested the requirement for the molecular diagnosis of nodal metastasis.

\section{References}

1. Eagon JC, Miedema BW and Kelly KA: Postgastrectomy syndromes. Surg Clin North Am 72: 445-465, 1992.

2. Morton DL, Wen DR, Wong JH, Economou JS, Cagle LA, Storm FK, Foshag LJ and Cochran AJ: Technical details of intraoperative lymphatic mapping for early stage melanoma. Arch Surg 127: 392-399, 1992.

3. Kitagawa Y, Takeuchi H, Takagi Y, Natsugoe S, Terashima M, Murakami N, Fujimura T, Tsujimoto H, Hayashi H, Yoshimizu N, et al: Sentinel node mapping for gastric cancer: A prospective multicenter trial in Japan. J Clin Oncol 31: 3704-3710, 2013.

4. Miwa K, Kinami S, Taniguchi K, Fushida S, Fujimura T and Nonomura A: Mapping sentinel nodes in patients with early-stage gastric carcinoma. Br J Surg 90: 178-182, 2003.

5. Kinami S, Fujimura T, Ojima E, Fushida S, Ojima T, Funaki H, Fujita H, Takamura H, Ninomiya I, Nishimura G, et al: PTD classification: Proposal for a new classification of gastric cancer location based on physiological lymphatic flow. Int J Clin Oncol 13: 320-329, 2008.

6. Miwa K: Sentinel node concept and its application for cancer surgery. Nihon Geka Gakkai Zasshi 101: 307-310, 2000 (In Japanese).

7. Hiratsuka M, Miyashiro I, Ishikawa O, Furukawa H, Motomura K, Ohigashi H, Kameyama M, Sasaki Y, Kabuto T, Ishiguro S, et al: Application of sentinel node biopsy to gastric cancer surgery. Surgery 129: 335-340, 2001.
8. Carlini M, Carboni F, Petric M, Santoro R, Guadagni F, Marandino F, Castelli M and Santoro E: Sentinel node in gastric cancer surgery. J Exp Clin Cancer Res 21: 469-473, 2002.

9. Ichikura T, Morita D, Uchida T, Okura E, Majima T, Ogawa T and Mochizuki H: Sentinel node concept in gastric carcinoma. World J Surg 26: 318-322, 2002.

10. Kitagawa Y, Fujii H, Mukai M, Kubota T, Otani Y and Kitajima M: Radio-guided sentinel node detection for gastric cancer. Br J Surg 89: 604-608, 2002.

11. Miyashiro I, Hiratsuka M, Sasako M, Sano T, Mizusawa J, Nakamura K, Nashimoto A, Tsuburaya A and Fukushima N; Gastric Cancer Surgical Study Group (GCSSG) in the Japan Clinical Oncology Group (JCOG): High false-negative proportion of intraoperative histological examination as a serious problem for clinical application of sentinel node biopsy for early gastric cancer: Final results of the Japan Clinical Oncology Group multicenter trial JCOG0302. Gastric Cancer 17: 316-323, 2014.

12. Wang Z, Dong ZY, Chen JQ and Liu JL: Diagnostic value of sentinel lymph node biopsy in gastric cancer: A meta-analysis. Ann Surg Oncol 19: 1541-1550, 2012.

13. Nimura H, Narimiya N, Mitsumori N, Yamazaki Y, Yanaga K and Urashima M: Infrared ray electronic endoscopy combined with indocyanine green injection for detection of sentinel nodes of patients with gastric cancer. Br J Surg 91: 575-579, 2004.

14. Kusano M, Tajima Y, Yamazaki K, Kato M, Watanabe M and Miwa M: Sentinel node mapping guided by indocyanine green fluorescence imaging: A new method for sentinel node navigation surgery in gastrointestinal cancer. Dig Surg 25: 103-108, 2008.

15. Tajima Y, Murakami M, Yamazaki K, Masuda Y, Kato M, Sato A, Goto S, Otsuka K, Kato T and Kusano M: Sentinel node mapping guided by indocyanine green fluorescence imaging during laparoscopic surgery in gastric cancer. Ann Surg Oncol 17: 1787-1793, 2010.

16. Kelder W, Nimura H, Takahashi N, Mitsumori N, van Dam GM and Yanaga K: Sentinel node mapping with indocyanine green (ICG) and infrared ray detection in early gastric cancer: An accurate method that enables a limited lymphadenectomy. Eur J Surg Oncol 36: 552-558, 2010.

17. Lee YJ, Ha WS, Park ST, Choi SK, Hong SC and Park JW: Which biopsy method is more suitable between a basin dissection and pick-up biopsy for sentinel nodes in laparoscopic sentinel-node navigation surgery (LSNNS) for gastric cancer? J Laparoendosc Adv Surg Tech A 18: 357-363, 2008.

18. Aoyama K, Kamio T, Ohchi T, Nishizawa M and Kameoka S: Sentinel lymph node biopsy for breast cancer patients using fluorescence navigation with indocyanine green. World J Surg Oncol 9: 157, 2011.

19. Crane LM, Themelis G, Arts HJ, Buddingh KT, Brouwers AH, Ntziachristos V, van Dam GM and van der Zee AG: Intraoperative near-infrared fluorescence imaging for sentinel lymph node detection in vulvar cancer: First clinical results. Gynecol Oncol 120: 291-295, 2011.

20. Fujisawa $Y$, Nakamura Y, Kawachi $Y$ and Otsuka F: Indocyanine green fluorescence-navigated sentinel node biopsy showed higher sensitivity than the radioisotope or blue dye method, which may help to reduce false-negative cases in skin cancer. J Surg Oncol 106: 41-45, 2012.

21. Imai K, Minamiya Y, Saito H, Nakagawa T, Ito M, Ono T, Motoyama S, Sato Y, Konno H and Ogawa J: Detection of pleural lymph flow using indocyanine green fluorescence imaging in non-small cell lung cancer surgery: A preliminary study. Surg Today 43: 249-254, 2013.

22. Ishizuka M, Nagata H, Takagi K, Iwasaki Y and Kubota K: Fluorescence imaging visualizes three sets of regional lymphnodes in patients with lower rectal cancer. Hepatogastroenterology 59: 1381-1384, 2012

23. Miyashiro I, Miyoshi N, Hiratsuka M, Kishi K, Yamada T, Ohue M, Ohigashi H, Yano M, Ishikawa $\mathrm{O}$ and Imaoka S: Detection of sentinel node in gastric cancer surgery by indocyanine green fluorescence imaging: Comparison with infrared imaging. Ann Surg Oncol 15: 1640-1643, 2008.

24. Rossi EC, Ivanova A and Boggess JF: Robotically assisted fluorescence-guided lymph node mapping with ICG for gynecologic malignancies: A feasibility study. Gynecol Oncol 124: 78-82, 2012.

25. Schaafsma BE, van der Vorst JR, Gaarenstroom KN, Peters AA, Verbeek FP, de Kroon CD, Trimbos JB, van Poelgeest MI, Frangioni JV, van de Velde CJ and Vahrmeijer AL: Randomized comparison of near-infrared fluorescence lymphatic tracers for sentinel lymph node mapping of cervical cancer. Gynecol Oncol 127: 126-130, 2012. 
26. Yoshida M, Kubota K, Kuroda J, Ohta K, Nakamura T, Saito J, Kobayashi M, Sato T, Beck Y, Kitagawa Y and Kitajima M: Indocyanine green injection for detecting sentinel nodes using color fluorescence camera in the laparoscopy-assisted gastrectomy. J Gastroenterol Hepatol 27 (Suppl 3): S29-S33, 2012.

27. Kubota K, Yoshida M, Kuroda J, Okada A, Ohta K and Kitajima M: Application of the HyperEye medical system for esophageal cancer surgery: A preliminary report. Surg Today 43 : 215-220, 2013

28. Tajima Y, Yamazaki K, Masuda Y, Kato M, Yasuda D, Aoki T, Kato T, Murakami M, Miwa M and Kusano M: Sentinel node mapping guided by indocyanine green fluorescence imaging in gastric cancer. Ann Surg 249: 58-62, 2009.

29. Japanese Gastric Cancer Association. Japanese classification of gastric carcinoma: III English edition. Gastric Cancer 14: 101-112, 2011.

30. Japanese Gastric Cancer Association. Japanese gastric cancer treatment guidelines 2010 (ver. 3). Gastric Cancer 14: 113-123, 2011.

31. Maruyama K, Sasako M, Kinoshita T, Sano T and Katai H: Can sentinel node biopsy indicate rational extent of lymphadenectomy in gastric cancer surgery? Fundamental and new information on lymph-node dissection. Langenbecks Arch Surg 384: 149-157, 1999.

32. Sirop S, Kanaan M, Korant A, Wiese D, Eilender D, Nagpal S, Arora M, Singh T and Saha S: Detection and prognostic impact of micrometastasis in colorectal cancer. J Surg Oncol 103 534-537, 2011.

33. Giuliano AE, Haigh PI, Brennan MB, Hansen NM, Kelley MC, Ye W, Glass EC and Turner RR: Prospective observational study of sentinel lymphadenectomy without further axillary dissection in patients with sentinel node-negative breast cancer. J Clin Oncol 18: 2553-2559, 2000.

34. Songun I, Putter H, Kranenbarg EM, Sasako M and van de Velde CJ: Surgical treatment of gastric cancer: 15-year follow-up results of the randomized nationwide Dutch D1D2 trial. Lancet Oncol 11: 439-449, 2010.

35. Brouwer OR, Buckle T, Vermeeren L, et al: Comparing the hybrid fluorescent-radioactive tracer indocyanine green-99mTc-nanocolloid with $99 \mathrm{mTc}$-nanocolloid for sentinel node identification: A validation study using lymphoscintigraphy and SPECT/CT. J Nucl Med 53: 1034-1040, 2012.
36. Frontado LM, Brouwer OR, van den Berg NS, Mathéron HM, Vidal-Sicart S, van Leeuwen FW and Valdés Olmos RA: Added value of the hybrid tracer indocyanine green-99mTc-nanocolloid for sentinel node biopsy in a series of patients with different lymphatic drainage patterns. Rev Esp Med Nucl Imagen Mol 32: 227-233, 2013.

37. Heuveling DA, Visser GW, de Groot M, de Boer JF, Baclayon M, Roos WH, Wuite GJ, Leemans CR, de Bree R and van Dongen GA: Nanocolloidal albumin-IRDye $800 \mathrm{CW}$ : A near-infrared fluorescent tracer with optimal retention in the sentinel lymph node. Eur J Nucl Med Mol Imaging 39: 1161-1168, 2012.

38. Kong SH, Noh YW, Suh YS, Park HS, Lee HJ, Kang KW, Kim HC, Lim YT and Yang HK: Evaluation of the novel near-infrared fluorescence tracers pullulan polymer nanogel and indocyanine green $/ \gamma$-glutamic acid complex for sentinel lymph node navigation surgery in large animal models. Gastric Cancer 18: 55-64, 2015.

39. Toyota T, Fujito H, Suganami A, Ouchi T, Ooishi A, Aoki A, Onoue K, Muraki Y, Madono T, Fujinami M, et al: Near-infrared-fluorescence imaging of lymph nodes by using liposomally formulated indocyanine green derivatives. Bioorg Med Chem 22: 721-727, 2014.

40. Takeuchi H, Ueda M, Oyama T, Shimizu Y and Kitagawa Y: Molecular diagnosis and translymphatic chemotherapy targeting sentinel lymph nodes of patients with early gastrointestinal cancers. Digestion 82: 187-191, 2010.

41. Yaguchi Y, Sugasawa H, Tsujimoto H, Takata H, Nakabayashi K, Ichikura T, Ono S, Hiraki S, Sakamoto N, Horio T, et al: One-step nucleic acid amplification (OSNA) for the application of sentinel node concept in gastric cancer. Ann Surg Oncol 18: 2289-2296, 2011. 